\title{
Detection and quantification of antibiotic residues in urine samples of healthy individuals from rural and urban communities in Ghana using a validated SPE-LC-MS/MS method
}

\author{
Samuel Oppong Bekoe ${ }^{1}$ - Emmanuel Orman ${ }^{2}$ (D) Samuel Asare-Nkansah ${ }^{1} \cdot$ Anne Mette Lerbech Sørensen ${ }^{3}$. \\ Erland Björklund $^{4}\left(\mathbb{D} \cdot\right.$ Reimmel Kwame Adosraku $^{1} \cdot$ Martin Hansen $^{3,5}\left([) \cdot\right.$ Bjarne Styrishave $^{3}([)$
}

Received: 27 July 2020 / Accepted: 19 October 2020 / Published online: 27 October 2020

(c) Springer Nature Switzerland AG 2020, corrected publication 2020

\begin{abstract}
The role of unregulated and inappropriate dispensing, and use of antibiotics remains significant in the development of antimicrobial resistance in infectious disease endemic regions of developing countries. The exposure to antibiotics from unfamiliar and unsuspecting sources such as drinking water and food, and adulterated herbal medicines remains a cause for concern. A sensitive SPE-LC-MS/MS method was developed and validated for the quantification and qualification of 12 antibiotics, including amoxicillin, clavulanic acid, metronidazole, ampicillin, cefuroxime, tetracycline, ceftriaxone, sulphamethoxazole, trimethoprim, ciprofloxacin, benzylpenicillin, and erythromycin, in the urine of healthy volunteers. The method was linear $\left(r^{2}>0.98\right)$ within the concentration range $50-5000 \mathrm{ngmL}^{-1}$ for all the analytes. Instrument precision of $8-27 \%$ and $4-21 \%$ at 100 and $1000 \mathrm{ngmL}^{-1}$ levels were demonstrated. High mean recoveries between 71 and $125 \%$ with minimal variations were obtained for all compounds in the accuracy study. Limits of detection and quantification ranged between $70.3-271.0 \mathrm{ngmL}^{-1}$ and $213-821 \mathrm{ngmL}^{-1}$ respectively. The validated method successfully detected and quantified 9 of the 12 analytes, with the exception of clavulanic acid, ceftriaxone, and benzylpenicillin. Most of the samples contained one analyte $(52,86.7 \%)$, with a handful containing two $(7,11.7 \%)$ and three analytes $(1,1.7 \%)$. Ciprofloxacin was the modal analyte detected $(17,24.6 \%)$, with amoxicillin and trimethoprim recording the average lowest $\left(22.76 \times 10^{3} \mathrm{ngmL}^{-1}\right)$ and highest concentrations $\left(255.47 \times 10^{3} \mathrm{ngmL}^{-1}\right)$ respectively. The developed method is a useful tool for non-invasive monitoring of consumption and the irrational use of antibiotics in microbial resistant-prone regions of the world.
\end{abstract}

Keywords Antibiotic residues · Antimicrobial resistance · Solid phase extraction (SPE) · Urine samples · Antibiotic exposure $\cdot$ LC-MS/MS method

Electronic supplementary material The online version of this article (https://doi.org/10.1007/s42452-020-03742-7) contains supplementary material, which is available to authorized users.

$\triangle$ Samuel Oppong Bekoe, sobek03@gmail.com; sobekoe.pharm@knust.edu.gh; Emmanuel Orman, eorman@uhas.edu.gh; Samuel Asare-Nkansah, asn12002@yahoo.com; Anne Mette Lerbech Sørensen, am_lerbech@hotmail.com; Erland Björklund, erland.bjorklund@ hkr.se; Reimmel Kwame Adosraku, etorwu@yahoo.com; Martin Hansen, martin.hansen@envs.au.dk; Bjarne Styrishave, bjarne.styrishave@ sund.ku.dk| ${ }^{1}$ Department of Pharmaceutical Chemistry, Faculty of Pharmacy and Pharmaceutical Sciences, Kwame Nkrumah University of Science and Technology, Kumasi, Ghana. ${ }^{2}$ Department of Pharmaceutical Chemistry, School of Pharmacy, University of Health and Allied Sciences, Ho, Ghana. ${ }^{3}$ Department of Pharmacy, Faculty of Health and Medical Sciences, University of Copenhagen, Universitetsparken 2, DK-2100 Copenhagen, Denmark. ${ }^{4}$ Division of Natural Sciences, School of Education and Environment, Kristianstad University, 29188 Kristianstad, Sweden. ${ }^{5}$ Department of Environmental Sciences-Environmental Chemistry and Toxicology, Aarhus University, Aarhus, Denmark. 


\section{Introduction}

The burden of infectious diseases resulting from resistant strains of pathogens contributes significantly to mortality rates in developing and low middle income countries [1]. Although limited evidence exist to indicate a direct correlation between antibiotic use and antimicrobial resistance [2], the link between sub-therapeutic amounts/residues of antimicrobial agents and the development of resistance by pathogens can also not be overruled $[3,4]$.

Prescribing patterns and misuse of antibiotics by health professionals, patients and unsuspecting healthy consumers play significant roles in the observed escalating trends of antimicrobial resistance issues globally [5, 6]. However, very little scientific information exist on the use or consumption patterns of these life-saving agents, especially in some low income to lower middle-income countries, where there is limited access to information or data from public health facilities. These may be as a result of factors like poor research capacity, poor documentation and storage, and poor information retrieval systems [7]. In addition, increased availability of antibiotics from unapproved sources, environmental residues, substandard products in the supply chain and patient non-adherence to prescribed doses of antibiotics, leading to inadequate systemic amounts of the medicinal agents, contribute to the observed trends of microbial resistance [8].

Studies have shown that the use and consumption of antibiotics could be influenced by factors that are socio-economic and behavioural in nature $[9,10]$. In countries with weak regulatory and law enforcement machinery, a patient or consumer with high purchasing power could request for antibiotics without prescription from a facility (regulated or un-regulated/approved or unapproved), leading to misuse and abuse of antibiotics [11]. Altogether, poor quality antibiotics [12-15], irrational prescribing, increasing self-medication practices by patients, and consumption of residues of antibiotics from food, really make it difficult for public health and clinical efforts to address the problem of antimicrobial resistance in developing and emerging countries [16, 17]. In Ghana for example, the reports of few studies on self-medication practices indicate high prevalence rates of self-medication with antibiotics among sections of the population $[18,19]$.

In an infectious disease endemic region such as Africa, antibiotics belonging to several different pharmacologic classes, are prescribed or inappropriately used or abused annually [20-22]. However, data on usage or prescription pattern is scanty, and when such scientific data or information is available, it is usually too erratic to allow for proper trend analysis. The usage trends normally appear heterogeneous in nature, varying from country to country [21] as well as from facility to facility (even within the same country). As a result, monitoring of the usage of antibiotics, and the planning and implementation of antibiotic resistant intervention programmes remain a challenge in several countries in Africa. The problem has further been complicated by the exposure of consumers unknowingly to sub-therapeutic doses of antibiotics from herbal medicine products and other nutraceuticals as a result of willful adulteration [23]. Very few drug regulatory laboratories on the African continent have both the technical and human capacity to consistently monitor the quality of antibiotics in the supply chain of the health delivery systems as well as access the levels of sub-therapeutic drug exposures from contaminations and adulterations. One key contribution to this is in limitations with respect to sensitive analytical methods and efficient sample preparation techniques to monitor the pre-and post-registration quality of antibiotics; an index that has been strongly argued as a determinant in the scheme of affairs for the development of microbial resistance $[3,4]$.

A number of liquid chromatographic analytical methods using different detectors (ultraviolet-visible, florescence spectroscopy and mass spectrometry) have been reported in literature for the identification and quantification of different antibiotics in various matrices. These methods are shown to be suitable for either single compound determination or simultaneous determination of up to six compounds in a sample [24-27]. However, very few of such studies have focused and reported on the determination of antibiotics excreted in urine, and urine samples spiked with desired analytes [28] possibly because of challenges with sample clean-ups. Sample clean-ups are reportedly achieved by liquid-liquid extraction or solid phase extraction (SPE) procedures [29]. SPE, however, has been the preferred procedure when biological matrices such as urine are involved, but only small volumes of samples $(20 \mathrm{~mL})$ could be handled at a time [30].

Due to the burden of life-threatening infections and the presence of myriad of antibiotics on the Ghanaian market, the current study focused on the development of a cost-effective and sensitive 'one-pot' chemical analytical method to monitor the extent of exposure of antibiotics from selected sites in Ghana through the analyses of urine samples of healthy volunteers. For this purpose, the study aimed at developing and validating a hyphenated liquid chromatography-mass spectrometry (LC-MS/ MS) method to determine simultaneously, 12 antibiotics (commonly prescribed, used and consumed) in human urine samples, using SPE for sample clean-up. The SPE 
procedure was also investigated and optimized to handle larger volumes of samples per run. It was further predicted that the method could be useful in bioanalytical studies involving determination of the systemic availability of antibiotics using biological matrices such as urine, saliva and serum.

\section{Experimental and methods}

\subsection{Reagents, standards and materials}

An analytical standard of amoxicillin was purchased from Duchefa Biochemie B.V (Netherlands). Ampicillin (AMP), metronidazole (MTZ), cefuroxime (CFX), ciprofloxacin (CPC), trimethoprim (TMP), and sulfamethoxazole (STX) were purchased from Fluka (Glostrup, Denmark). Tetracycline hydrochloride (TCC) and erythromycin (ETM) were purchased from Sigma-Aldrich, (Glostrup, Denmark). The internal standard (IS) $d_{4}$-sulfamethoxazole was purchased from Toronto Research Chemicals (TRC), and $\mathrm{d}_{3}$-trimethoprim and $\mathrm{d}_{8}$-ciprofloxacin were obtained from Qmx Laboratories (Essex, UK). All analytical standards had a purity above $99 \%$. Acetonitrile and methanol were of HPLC grade and purchased from Lab-Scan. Ammonium formate $(\geq 99.0 \%)$, formic acid $(98 \%)$, and triethylamine $(\geq 99 \%)$ were purchased from Merck KGaA (Darmstadt, Germany), and Sigma-Aldrich (Seelze, Germany), respectively. Milli-Q water gradient system (Millipore, Bedford, MA, USA) was used in all analyses. Solid phase extractions were performed on Oasis ${ }^{\circ} \mathrm{HLB} 6 \mathrm{~cm}^{3} 200 \mathrm{mg}(30 \mu \mathrm{m})$ cartridges from Waters (Milford, MA, USA). The vacuum manifold was an IST Vacmaster from Biotage (Uppsala, Sweden), and the pump was a VacSafe 15, from ScanLaf A/S (Lynge, Denmark). The $\mathrm{pH}$ of the urine samples was measured by a CyberScan pH 510 from Eutech Instruments (Germany). The evaporator used was a Dionex SE 500 (CA, USA).

\subsection{Standard solutions}

A standard antibiotic-mix with a concentration at approximately 10 ppm was prepared by mixing known amount of each stock solution with methanol in a $10 \mathrm{~mL}$ volumetric flask. Likewise, an internal standard solution with a concentration at approximately $10 \mathrm{ppm}$ for each IS was prepared. The calibration concentration range (in HPLC-vial) was $50-5000 \mathrm{ngmL}^{-1}$ (working concentration range) for all analytes and $500 \mathrm{ngmL}^{-1}$ for IS. The standard solutions were protected from light and stored at $-18^{\circ} \mathrm{C}$. Working concentrations were prepared by appropriate dilutions with mobile phase $A: B(10: 90, v / v)$.

\subsection{Sampling of human urine from healthy volunteers}

Urine samples were obtained from 177 healthy subjects; 113 from Kumasi, an urban community; and 64 from Awenare, a rural community. Households within the submetro districts in the Kumasi metropolis and Awenare were visited and residents were briefed and when written consent [S1] were given, subjects provided urine samples using prescribed containers. Sampling was undertaken during the period between July and October, 2012. Volunteers who were on prescribed antibiotics for 2 weeks prior to the study or were knowingly taking any antibiotics by themselves were excluded from the study. Subjects who needed a drink to facilitate their urine flow were each given a bottle of water $(500 \mathrm{~mL})$.

\subsection{Sample preparation}

Urine samples were collected into prescribed containers, labeled and weighed. This was followed by the addition of $100 \mu \mathrm{L} \mathrm{IS}\left(10 \mu \mathrm{gmL}^{-1}\right)$ and $100 \mu \mathrm{L} 0.01 \mathrm{M}$ citrate buffer to each sample. The IS adopted were selected because they possessed similar physicochemical properties to the compounds of interest when in solution. Furthermore, d4-Sulphamethoxazole was chosen to serve as IS for the analysis of analytes in the negative ionization mode whiles d3-trimethoprim and d8-ciprofloxacin were selected to serve as IS for analysis in the positive ionization mode.

The $\mathrm{pHs}$ of the samples were measured and adjusted when necessary with $2 \mathrm{M} \mathrm{H}_{2} \mathrm{SO}_{4}$ or $2 \mathrm{M} \mathrm{NaOH}$ to $7.0 \pm 0.3$. Samples were then frozen at $-18{ }^{\circ} \mathrm{C}$ pending extraction, unless samples were extracted the day they were obtained. Urine samples were enriched using SPE with Oasis ${ }^{\circ}$ HLB $6 \mathrm{~cm}^{3} 200 \mathrm{mg}(30 \mu \mathrm{m})$ cartridges from Waters (Milford, MA, USA). The HLB cartridges (polymeric resins) were chosen because their lipophilic and hydrophilic retention characteristics enhance the retention of a wide spectrum of both polar and non-polar compounds, and this has been well established for the compounds under study [30]. Before use, the cartridges were conditioned with $2 \mathrm{~mL}$ methanol followed by $2 \mathrm{~mL} 0.01 \mathrm{M}$ citrate buffer $(\mathrm{pH} 7 \pm 0.2)$ and $2 \mathrm{~mL}$ distilled water. Urine samples (averagely $110 \mathrm{~mL}$ ) were loaded on to the SPE columns with a flow rate of 1-1.5 mL/ min. After the extraction, the sorbent was carefully dried under vacuum for up to an hour. The SPE columns were thereafter stored at $-18^{\circ} \mathrm{C}$, and later transported to Denmark for the washing and elution of antibiotic compounds for analysis. During the transport of the SPE cartridges, all necessary cold chain conditions were ensured. Moreover, the contents of internal standards (serving as quality control standards) added were analyzed to establish whether degradation had occurred during transport. The sorbent 
was washed with $3 \mathrm{~mL}$ methanol and dried under vacuum for 5-10 min. Compounds were eluted using a $5 \mathrm{~mL} \mathrm{mix-}$ ture of mobile phase $A: B(10: 90, v / v)$ and $3 \mathrm{~mL}$ methanol: water $(80: 20, \mathrm{v} / \mathrm{v})$. The developed $\mathrm{LC}$ method consisted of a gradient of the mobile phases $A$ and $B$. Mobile phase A was water: acetonitrile (99.8:0.2, $\mathrm{v} / \mathrm{v})$ and that of $B$ was water: acetonitrile $(5: 95, v / v)$. Both mobile phases contained $100 \mu \mathrm{L}$ formic acid (98\%) and $100 \mu \mathrm{L}$ triethylamine per liter. The extracts were evaporated to dryness under a gentle stream of nitrogen gas at $30^{\circ} \mathrm{C}$ and re-dissolved in $200 \mu \mathrm{L}$ of mobile phase $A: B(1: 1)$. Solutions were subjected to LC-MS/MS analysis.

\subsection{LC-MS/MS analysis}

LC-MS/MS analyses were performed on all urine samples obtained using the mobile phase system described under Sect. 2.4. The HPLC system consisted of a binary pump, a degasser, a cooled auto sampler $\left(4^{\circ} \mathrm{C}\right)$, an injector $(10 \mu \mathrm{L})$ and a column oven $\left(30^{\circ} \mathrm{C}\right)$. The column stationary phase was Waters XTerra MS $\mathrm{C}_{18}(2.1 \times 100 \mathrm{~mm}$; $2.5 \mu \mathrm{m})$ and an equivalent $10 \mathrm{~mm}$ guard column. Flow rate and injection volume were $250 \mu \mathrm{Lmin}^{-1}$ and $5 \mu \mathrm{L}$ respectively. The developed LC method consisted of a gradient of the mobile phases $A$ and $B$, starting with $1 \%$ (B) holding for $5 \mathrm{~min}(0-5 \mathrm{~min})$ and increasing to $75 \%$ (B) during $15 \mathrm{~min}$ (5-20 min). Isocratic elution at $75 \%$ (B) followed for another minute (20-21 min). Mobile phase A was increased back to $99 \%$ within $1 \mathrm{~min}(21-22 \mathrm{~min})$ and equilibrated for another $8 \mathrm{~min}$ (22-30 min). The compound specific parameters optimised for the different compounds are as given in Table 1. MS detection was performed using Multiple Reaction Monitoring (MRM) setting. Precursor and product mass ions are listed in Table 1 as MRM ion pair.

\subsection{Matrix effect evaluation}

This effect was initially investigated qualitatively using post-column infusion experiments [31] to detect the presence of either suppression or enhancement on the compounds of interest. It was further evaluated using the spike-recovery experiments, where blank urine sample (without the analytes) was extracted using the developed SPE protocol, and the eluent spiked with defined amounts of the analytes prior to analysis. The outcome of this determination was compared with that of a blank mobile phase system, used in place of the blank urine sample.

\subsection{Validation}

The validation of analytical procedures for analytes determined in biological matrices is a pre-requisite for the development of selective and sensitive methods for the determination of such compounds. The method in this study was validated as per the guidelines of the International Council for Harmonisation [32, 33]. Selectivity of the method was demonstrated from uniqueness of the fragmentation patterns (the quantifier and qualifier) for each of the compounds. The ratio of the peak intensities of the quantifier to the qualifier of each analyte was characteristic of each compound and this was used selectively to detect and quantify them. The method was also

Table 1 Mass spectrometry specific parameters in the validated LC-MS/MS analytical method indicating ion transitions for 12 investigated antibiotics

\begin{tabular}{|c|c|c|c|c|c|c|c|c|c|}
\hline & Mode of ionization & Precursor ion & Quant > Qual. & Dwell time (MS) & $\mathrm{DP}(\mathrm{V})$ & $\mathrm{FP}(\mathrm{V})$ & $\mathrm{EP}(\mathrm{V})$ & $\mathrm{CE}(\mathrm{eV})$ & $\mathrm{CXP}(\mathrm{V})$ \\
\hline Amoxicillin & Negative & 364.3 & $222.8>302.8$ & 2000 & -50 & -200 & -10 & -20 & -10 \\
\hline Clavulanic acid & Negative & 197.9 & $135.7>123.9$ & 1200 & -30 & -100 & -5 & -10 & -20 \\
\hline Ampicillin & Negative & 348.1 & $206.8>172.9$ & 200 & -20 & -100 & -15 & -20 & -10 \\
\hline Metronidazole & Positive & 172.1 & $128.0>111.0$ & 500 & 20 & 50 & 10 & 20 & 15 \\
\hline Trimethoprim & Positive & 292.2 & $262.1>182.0$ & 200 & 30 & 150 & 10 & 30 & 10 \\
\hline d3-Trimethoprim & Positive & 294.1 & $264.1>184.1$ & 200 & 30 & 150 & 10 & 30 & 10 \\
\hline Tetracycline & Negative & 443.2 & $357.9>186.9$ & 200 & -20 & -200 & -10 & -30 & -10 \\
\hline Sulphamethoxazole & Negative & 252.0 & $155.7>108.2$ & 200 & -20 & -100 & -5 & -20 & -15 \\
\hline d4-Sulphamethoxazole & Negative & 256.0 & $159.7>111.1$ & 200 & -20 & -100 & -5 & -20 & -15 \\
\hline Cefuroxime & Negative & 423.2 & $317.8>206.7$ & 200 & -20 & -100 & -10 & -10 & -15 \\
\hline Ciprofloxacin & Positive & 332.6 & $288.0>245.0$ & 200 & 50 & 200 & 10 & 30 & 20 \\
\hline d8-Ciprofloxacin & Positive & 340.0 & $322.3>296.4$ & 200 & 50 & 200 & 10 & 30 & 20 \\
\hline Ceftriaxone & Positive & 555.4 & $396.1>352.0$ & 200 & 20 & 150 & 15 & 20 & 30 \\
\hline Penicillin G & Negative & 333.1 & $191.8>288.8$ & 200 & -30 & -100 & -10 & -10 & -10 \\
\hline Erythromycin & Positive & 734.2 & $158.3>576.3$ & 200 & 20 & 100 & 10 & 35 & 30 \\
\hline
\end{tabular}

KEY:DP declustering potential, FP focussing potential, EP entrance potential, CE collision energy, CXP collision cell exit potential 
validated for linearity, instrument precision and instrument accuracy. The limit of detection (LOD) and limit of quantification were also evaluated. Linearity was investigated using a 12-point calibration curve in the range of 50-5000 $\mathrm{ngmL}^{-1}$ for all analytes. Internal standard at concentration of $500 \mathrm{ngmL}^{-1}$ was added to all analyte solutions. Instrument limit of detection (LOD) and limit of quantitation (LOQ) were determined also in accordance with the guidelines $[32,33]$ The instrumental precision was investigated using neat standards at 100 and $1000 \mathrm{ngmL}^{-1}$ concentrations for all analytes. Instrument accuracy was determined by spiking potable water samples from the laboratory with neat standards at two concentration levels (100 and $1000 \mathrm{ngmL}^{-1}$ ). Validation of the overall SPE-LCMS/MS method was done at two concentration levels (100 and $1000 \mathrm{ngmL}^{-1}$ ) and recoveries were determined.

\section{Results and discussion}

\subsection{LC-MS/MS method development and optimization}

This LC-MS/MS method has been developed and validated to quantify individually as well as simultaneously, 12 antibiotic compounds in urine samples. The current method was modified and optimized from a previously reported method [34]. The modification involved the mobile phase system using various combinations of water and acetonitrile. A reverse phase gradient elution was finally selected consisting of mobile phase $A$ and mobile phase $B$ as described in Sect. 2.4. The binary gradient system was capable of maintaining the appropriate acid-base environment to ensure sufficient solubility of the compounds in the course of elution. This led to a sensitive and efficient analytical method which could separate the mixture of antibiotics with detection by electrospray ionization mass spectrometer (ESI) either in the positive or negative mode depending on the chemical architecture of the compounds. Compound specific or dependent parameters (Table 1; [S2], [S3]) for each compound was optimized, with at least two product ions obtained for each compound including the internal standards in order to minimise errors in the acquisition of data [35]. Precursor ions and fragmentation patterns (quantifier and qualifier) were recorded for each of the compounds. Precursor ions were mostly $[\mathrm{M}-\mathrm{H}]^{-}$ions and $[\mathrm{M}+\mathrm{H}]^{+}$ions for measurements in the negative and positive modes, respectively. Multiple Reaction Monitoring (MRM) setting was employed in all analyses to assure efficient separation and identification of the individual compounds. The developed LC-MS/ MS method was employed in all subsequent analyses of samples.

\subsection{Matrix effect, washing step and elution volume on recoveries}

From the post-column infusion experiment carried out (data not shown), the urine matrix was observed to have significant effect on peak intensities as well as instrumental efficiency. This phenomenon therefore informed the introduction and optimization of the washing step prior to elution. The final extraction procedure employing Oasis ${ }^{\circ}$ HLB $6 \mathrm{~cm}^{3} 200 \mathrm{mg}$ cartridges was thus optimized by investigating the washing and elution steps that preceded the LC-MS/MS analysis. Urine is a complex matrix to work with, due to its organic as well as inorganic contents. In order to evaluate matrix effect on the method, especially when large volumes of urine (over $100 \mathrm{~mL}$ ) are extracted, a washing step was investigated, optimized and incorporated in the SPE method. An optimized volume of $3 \mathrm{~mL}$ methanol was used for washing the cartridges prior to elution to further validate the removal of interferences from the urine matrix as shown in Fig. 1. The elution volume of $5 \mathrm{~mL}$ mobile phase A: B (10:90, v/v) followed by $3 \mathrm{~mL}$ methanol: water $(80: 20, v / v)$ as described in Sect. 2.4 was critical in ensuring optimized elution of antibiotics trapped on the sorbent. This volume was optimized to avoid possible losses of antibiotic compounds (Fig. 2). The recoveries for the extraction were determined by spiking blank urine samples $(200 \mathrm{~mL})$ with known concentrations of analytes and internal standards at two different concentration levels. The mean recoveries for the 12 antibiotic compounds as observed, at $100 \mathrm{ngmL}^{-1}$ and $1000 \mathrm{ngmL}^{-1}$ were therefore $90 \%$ and $95 \%$ respectively (Table 2 ). These critical steps allowed for a matrix effect of $5-10 \%$.

\subsection{Validation of LC-MS/MS method}

Calibration curves for all 12 analytes were obtained and good linearity was observed for concentrations in the range of $50-5000 \mathrm{ngmL}^{-1}$ (Table 2). Correlation coefficients $\left(r^{2}\right)$ were greater than 0.98 , and instrument LOD and LOQ for all analytes were in the range $70.3-271.0 \mathrm{ngmL}^{-1}$ and $213.1-821.2 \mathrm{ngmL}^{-1}$ respectively. Intra-day instrument precision (or repeatability) was investigated using six injections of standard solution at two different concentration levels on the calibration curves (Table 2). Precision expressed as RSD at two concentration levels were between 4.0 and $27.2 \%$. The results obtained indicated good precision at the two concentration levels, with the highest variability obtained at the lowest concentration level. Higher variabilities were also observed for compounds detected in the positive mode (that is, CTX, CPF, TMP and ETM) as compared to those detected in the negative mode (Table 2), and this could be attributed to the differences in instrumental sensitivity of the 


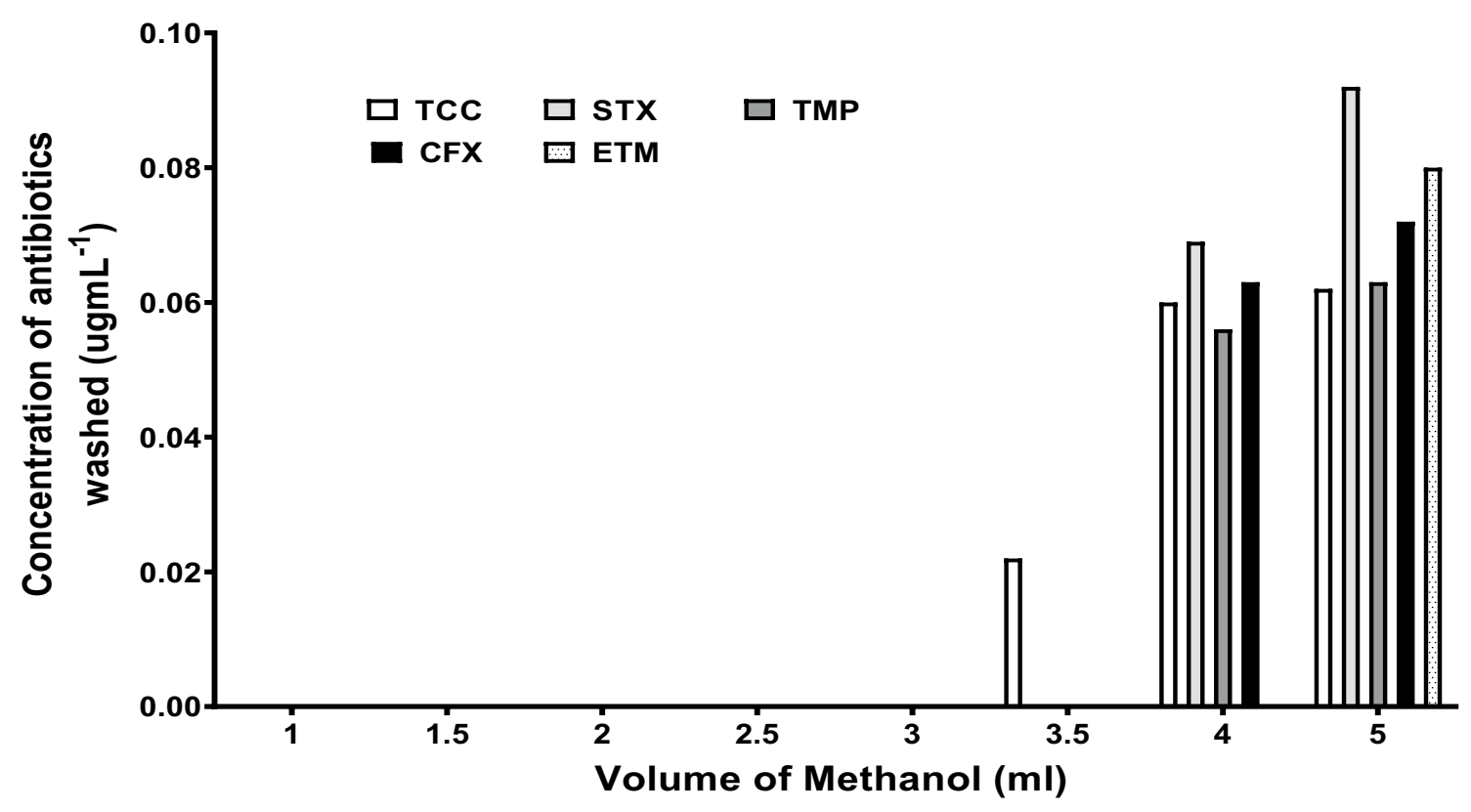

Fig. 1 Optimisation of the volume of methanol used for washing of the HLB cartridges $\left(6 \mathrm{~cm}^{3}, 200 \mathrm{mg}\right)$ prior to elution to ensure interference with urine matrix is minimal. No antibiotics were washed with 1-3 $\mathrm{mL}$ of methanol. A concentration of TCC was washed with $3.5 \mathrm{~mL}$ of methanol. Various concentrations of antibiotics were washed along with 4 and $5 \mathrm{~mL}$ of methanol

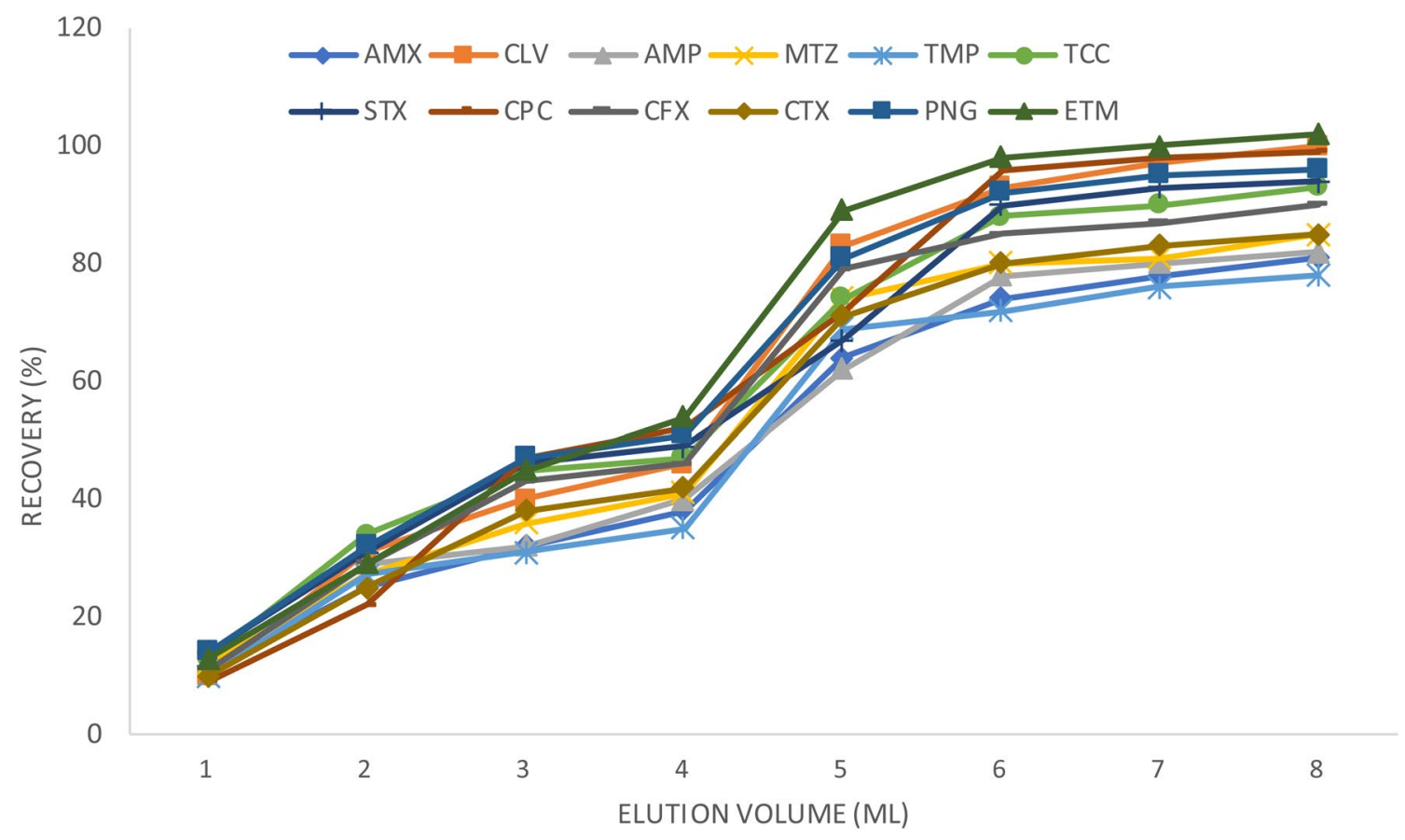

Fig. 2 Optimisation of the elution volume required to elute the 12 antibiotic compounds during solid phase extraction. The Oasis HLB $6 \mathrm{~cm}^{3}, 200 \mathrm{mg}$ cartridges were loaded with $200 \mathrm{~mL}$ spiked

affected analytes as a result of the MS ionization modes adopted. That notwithstanding, CTX, CPF, TMP and ETM all had very good signal-to-noise intensities. Minimal urine at a concentration level of $100 \mathrm{ngmL}^{-1}$ of each analyte. Each data point is a mean of duplicate determination

variabilities of corresponding high mean recoveries were also obtained for all compounds for the accuracy study. The mean recoveries were between 71 and 125\% (at the 


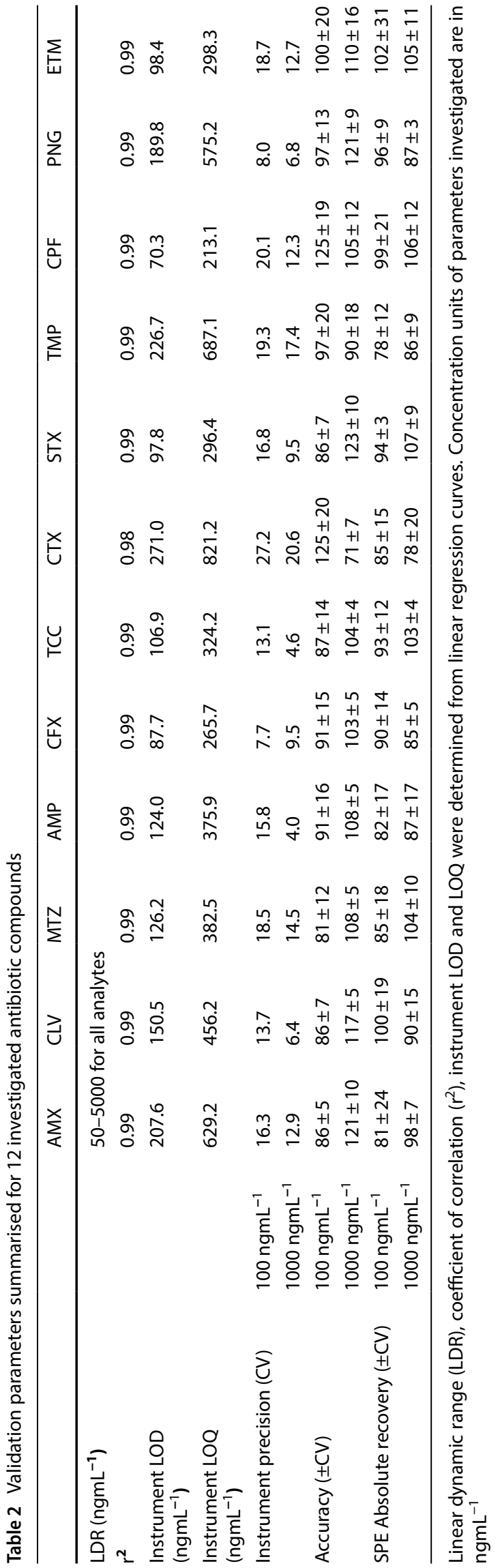

two concentration levels). Absolute recoveries for the SPE LC-MS/MS methodology were investigated at two concentration levels in spiked blank urine $\left(100 \mathrm{ngmL}^{-1}\right.$ and $1000 \mathrm{ngmL}^{-1}$ ). Recoveries obtained were between $78.3-102.5 \%$ and $77.9-106.7 \%$ respectively, with corresponding precision variations as RSD of $3-31 \%$ and $3-20 \%$ (Table 2). It is worth noting with the developed analytical method that, with the exception of TMP, CPC and CTX, the analytes generally had better accuracies at the higher concentration level compared to the lower level. Based on these data, the developed methodology for the identification and quantification of 12 antibiotics was applied to urine samples obtained from Ghana.

\subsection{Analysis of urine samples}

The developed method was applied to analysing large volumes of urine to determine the presence or otherwise of antibiotics and the results are discussed accordingly.

\subsubsection{Antibiotics detected in the urine samples?}

Nine out of the 12 target analytes (75.0\%) belonging to seven different therapeutic classes of antibiotics were detected in 36 (31.9\%) and 24 (37.5\%) of urines of the healthy volunteers from Kumasi metropolis and Awenare respectively (Fig. 3). Clavulanic acid (CLV), ceftriaxone (CTX) and benzyl penicillin (PNG) were not detected in any of the samples (Fig. 3). The absence of CLV in the urine samples may also indicate the lack of exposure of amoxicillin and clavulanic acid combination products to the volunteers (since the only source of CLV on the Ghanaian market is from such products). Comparing to AMX monotherapy, the combination product is relatively expensive and not easily accessible, hence may deter consumers from patronising them. CTX and PNG on the other hand may also not be easily accessible because of the parenteral administration route for PNG and cost of CTX, hence their absence in the test samples.

CPF $(17,24.6 \%), \operatorname{MTZ}(13,18.8 \%)$ and TCC $(11,15.9 \%)$ were among the frequently encountered antibiotics in the samples from both sites. Their detection in higher number of the urine samples may indicate their relative high patronage by the public [36-38], hence their exposure to the test subjects. Most of the urine samples $(52,86.7 \%)$ were shown to contain single analytes, with a few containing two analytes $(7,11.7 \%)$ and one contained three analytes (Fig. 4). For samples containing two analytes $(n=7)$, it was again observed that 4 (57.1\%) contained STX and TMP (which could be from the fixed dose combination medicines), with each of the other combinations $(n=3)$ containing TCC with either AMX, CFX and AMP. For the sample containing 

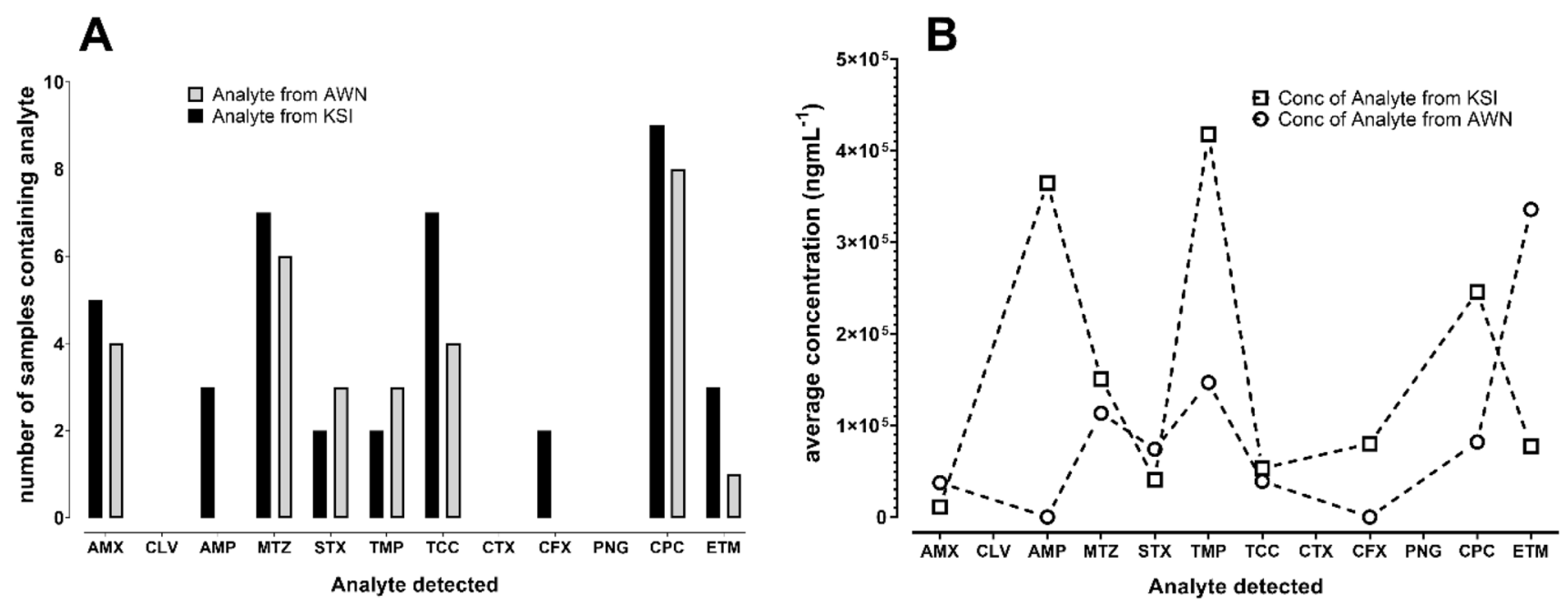

Fig. 3 Target analytes with their frequencies (a) and respective average concentrations detected (b) from the urine samples of healthy volunteers in Kumasi and Awenare
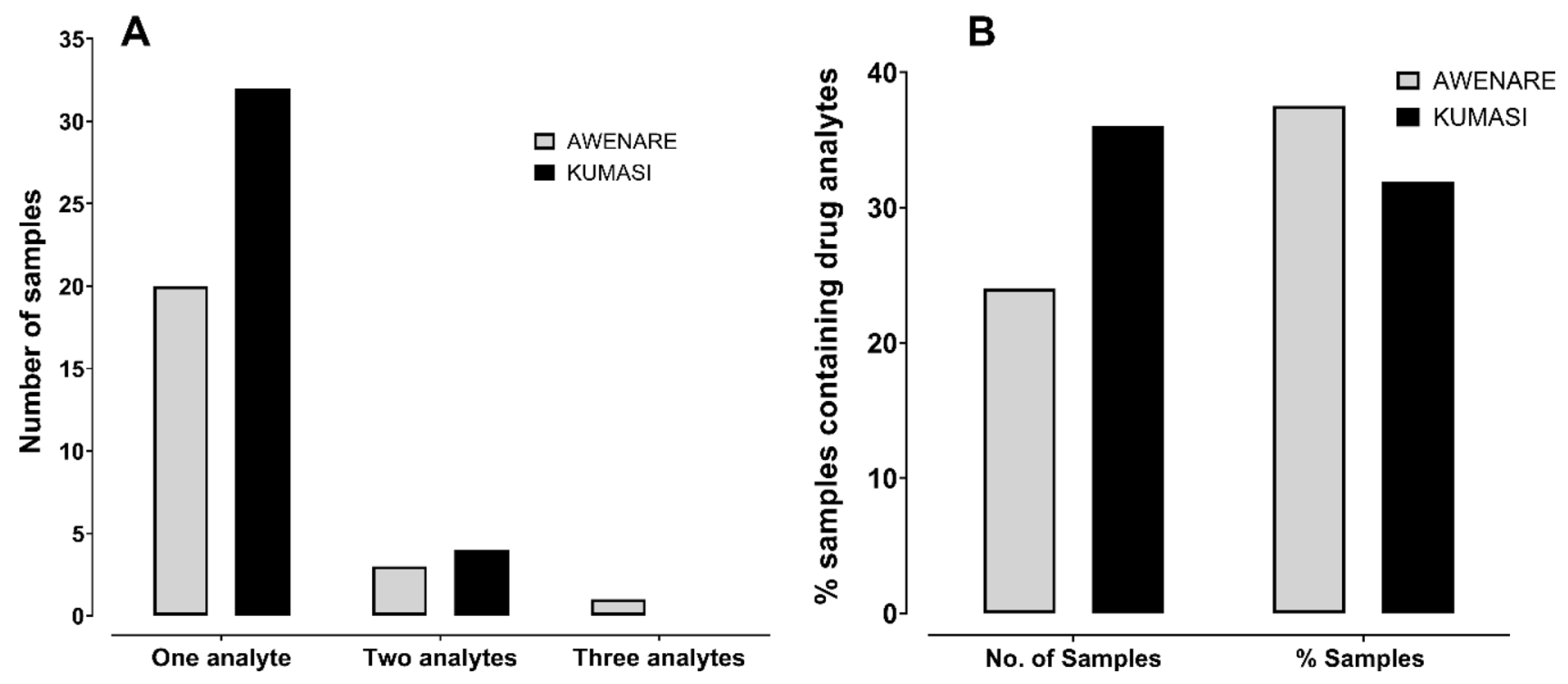

Fig. 4 Information on analytes detected in Urine samples. (a) Urine samples containing one or more antibiotic agent. Data representing raw values as from analyses. (b) Composition of urine samples analysed containing drug analytes. Data representing raw values as from analyses

three analytes, the combination was STX with TMP and CPF. Generally, TMP (Range: 77.51-666.99 $\times 10^{3} \mathrm{ngmL}^{-1}$ ), AMP (Range: $19.60-683.02 \times 10^{3} \mathrm{ngmL}^{-1}$ ) and CPC (25.56-846.95 $\left.\times 10^{3} \mathrm{ngmL}^{-1}\right)$ were amongst the detected analytes with very high concentrations (Fig. 5). The least concentration detected was for AMX. That notwithstanding, all the analytes' concentrations were thought to be comparable statistically $\left(F_{(8,60)}=1.870, p=0.0817\right)$ (Fig. 5).

\subsubsection{Rural-urban distribution of detected antibiotics}

The study showed some similarities and variations in the distribution of detected antibiotics. In terms of the number of urine samples analyzed, it was shown that the point prevalence of antibiotics was higher in the rural setting $(24,37.5 \%)$ than in the urban setting (36, $31.9 \%$ ) (Fig. 4b). This could be attributed to the less strict 


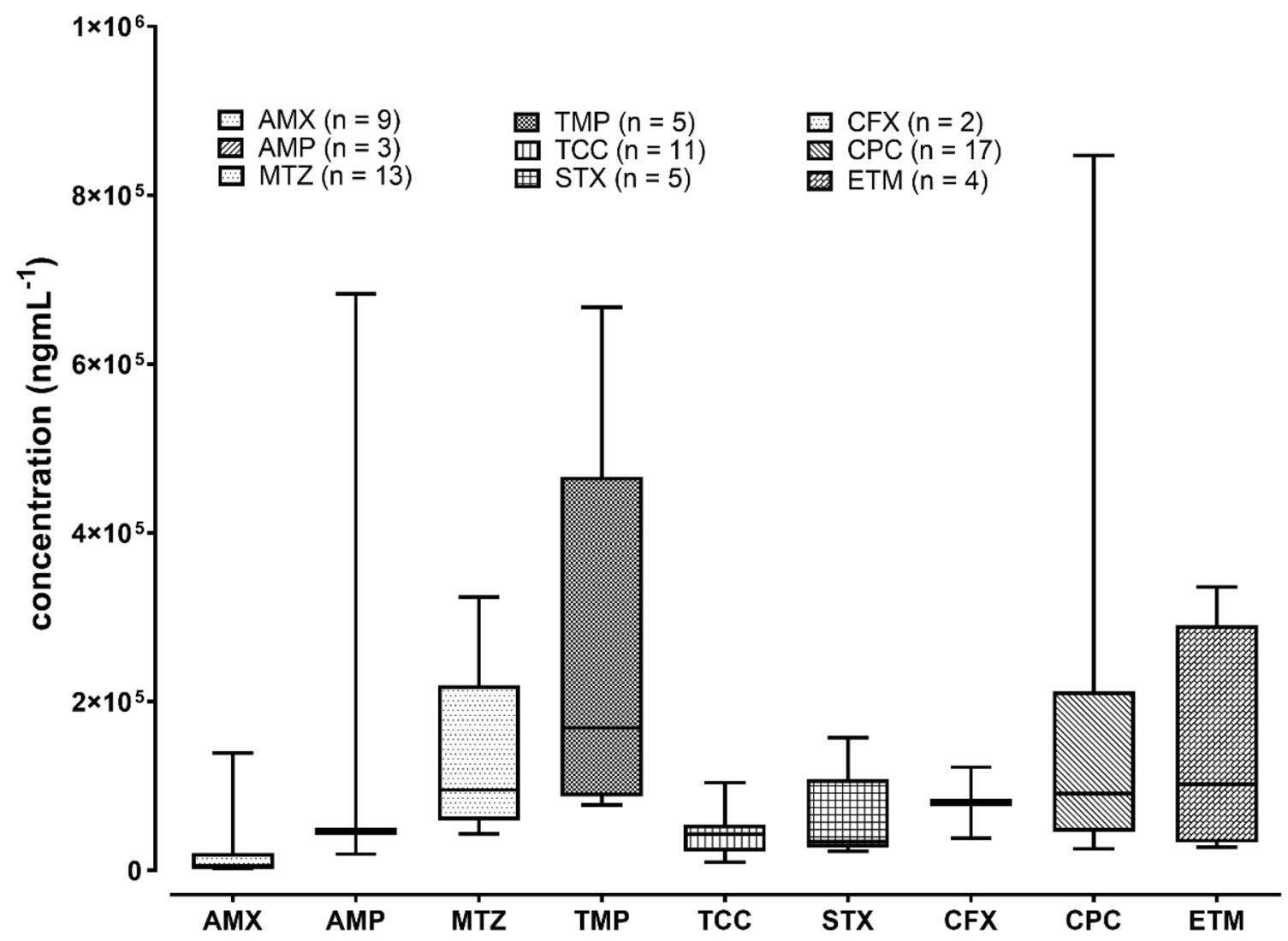

Fig. 5 Concentrations of analytes detected in the urine samples. One-Way ANOVA carried out showed that the concentrations of the analytes were comparable $\left(F_{8,60}=1.870 ; p=0.0817\right)$

regulation regarding the prescription, dispensing and the use of antibiotics in the rural setting as compared to the urban setting [39]. Some studies have demonstrated an inverse relationship between level of knowledge on antibiotics and their use especially among individuals in urban settings [40]. Out of the 9 analytes detected, 7 $(77.8 \%)$ were identified in samples from both sites, with 5 of them (71.4\%) occurring more in samples from Kumasi. On the other hand, STX and TMP, were observed more in samples from Awenare (Fig. 3). Previous studies had reported that co-trimoxazole (which is known to contain STX and TMP) were highly patronized in rural communities [37] and so it stands to reason then that individuals in such settings were more at risk of exposure to STX and TMP than to other antibiotics. Their high patronage has been attributed to their affordability, which in turn make them more favoured to be procured into both public and private facilities [37].

Two of the analytes, AMP and CFX, were only detected in samples from Kumasi. The relative higher occurrence of these antibiotics from the urban setting could as well be attributed to their wide availability, easy access and hence exposure to the volunteers. In addition, CFX is thought to be expensive and so better suited for use in the urban than the rural setting [41].

\subsubsection{Implications of the findings}

If the volunteers who participated in the study had really not taken antibiotics for at least 2 weeks prior to the study (as was required for inclusion in the study), then it is relevant to question the origins of the detected antibiotics. Probable sources could be from adulterated herbal medicines [23] as volunteers had indicated patronising herbal products during the survey [S1], water [42], and/ or food and veterinary products where lots of antibiotics are employed as growth promoters or therapeutic agents [43]. It could also be from unreported self-medication by the volunteers [19]. The public health implications of these findings cannot be overemphasized, as most of the detected antibiotics form part of essential medicines in Ghana [44] used either for primary or adjunct treatment of acute and potentially life-threatening conditions such as typhoid fever, gonorrhoea, bacterial diarrhoea, urinary tract infections, pelvic inflammatory diseases, liver abscess, $H$. pylori eradication, C. difficile infections, cholera, genital 
and respiratory mycoplasma infections [45]. As a result, the indiscriminate use and exposure to these antibiotics, as demonstrated by our study, may undermine both global and national efforts to maintain the integrity of life-saving antimicrobial agents by increasing the rate of microbial resistance through exposure of pathogens to their sublethal concentrations [46].

\section{Conclusion}

An optimised solid phase extraction procedure followed by LC-MS/MS method of quantification of antibiotics in large volume urine has been reported. The method which showed high accuracy and precision with high sensitivity was successfully applied to the simultaneous detection and quantitation of 12 antibiotics in urine samples of healthy volunteers. The study showed that significant residues of some of the essential antibiotics, were found in these volunteers and this may be a cause for worry as attempts made globally to fight antimicrobial resistance would be undermined by exposure of sub-lethal concentrations to the microorganisms.

Acknowledgements The authors are very grateful to the ADMER project management team, Ghana and Denmark. (http://admerproje ct.org/) and the Ministry of Health (MoH), Ghana for their support and guidance in the conduct of the research.

Authors' contribution All authors contributed equally to different parts of the study; including the study conception and design, material preparation, data collection and analysis, writing of the first draft of the manuscript, making inputs to revise the draft, reading and approval of the final manuscript.

Funding This study was funded by DANIDA, the Ministry of Foreign Affairs of Denmark, with a grant number-DFC number 09-099 SSI; Website: http://um.dk/en/danida-en/.

Availability of data and material The processed data used to support the findings of this study are included within the article. The written consent form used in recruiting volunteers is included as a supplementary data [S1]. Chromatograms showing the detection and quantitation of the antibiotics in positive mode [S2] and negative mode [S3] are also included as supplementary data.

\section{Compliance with ethical standards}

Conflict of interest Authors declare that they have no conflict of interest.

Ethical approval Ethical approval for the study (ID: CHRPE/65/11), was obtained from the Committee on Human Research, Publications and Ethics, KNUST School of Medical Sciences, and the Komfo Anokye Teaching Hospital (KATH), Kumasi, Ghana.

Consent for publication Not applicable.
Code availability Not applicable.

\section{References}

1. World Health Organization (2014) Antimicrobial resistance: global report on surveillance 2014. World Health Organization, Geneva, pp 1-257 ISBN: 9789241564748

2. Davies PDO (2004) Does increased use of antibiotics result in increased antibiotic resistance? Clin Infect Dis 39(1):18-19. https ://doi.org/10.1086/420826

3. Kelesidis T et al (2007) Counterfeit or substandard antimicrobial drugs: a review of the scientific evidence. J Antimicrob Chemother 60(2):214-236. https://doi.org/10.1093/jac/dkm109

4. Kelesidis T, Falagas ME (2015) Substandard/counterfeit antimicrobial drugs. Clin Microbiol Rev 28(2):443-464. https://doi. org/10.1128/CMR.00072-14

5. Attah CO, Aryee DN (2010) A study of antibiotic use and abuse in Ghana: a case study of the Cape Coast Metropolis. Internet J Health 11(2):5580. https://doi.org/10.5580/bec

6. Prah J et al (2017) Antibiotic prescription pattern in a Ghanaian primary health care facility. Pan Afr Med J 28:214. https://doi. org/10.11604/pamj.2017.28.214.13940

7. Yevutsey SK et al (2017) Situational analysis of antibiotic use and resistance in Ghana: policy and regulation. BMC Public Health 17(1):896. https://doi.org/10.1186/s12889-017-4910-7

8. Rozendaal J (2000) Fake antimalarials circulating in Cambodia. Bull Mekong Malaria 7:62-69 https://ci.nii.ac.jp/naid/10024 169084/. Accessed 5 Mar 2020

9. Hart CA, Kariuki S (1998) Antimicrobial resistance in developing countries. BMJ 317(7159):647-650. BMJ Publishing Group. https ://doi.org/10.1136/bmj.317.7159.647

10. Okeke IN, Lamikanra A, Edelman R (1999) Socioeconomic and behavioral factors leading to acquired bacterial resistance to antibiotics in developing countries. Emerg Infect Dis 5(1):18-27. Centers for Disease Control and Prevention (CDC). https://doi. org/10.3201/eid0501.990103

11. Van Den Boom GJM, Nsowah-Nuamah NNN, Overbosch GB (2008) Health-care provision \& self-medication in Ghana. In: The economy of Ghana, pp 392-416

12. Menkes DB (1997) Hazardous drugs in developing countries. BMJ 315(7122):1557-1558. BMJ Publishing Group. https://doi. org/10.1136/bmj.315.7122.1557

13. Pécoul B et al (1999) Access to essential drugs in poor countries: a lost battle? JAMA 281(4):361-367. https://doi.org/10.1001/ jama.281.4.361

14. Shakoor O, Taylor RB, Behrens RH (1997) Assessment of the incidence of substandard drugs in developing countries. Trop Med Int Health 2(9):839-845. https://doi. org/10.1046/j.1365-3156.1997.d01-403.x

15. Taylor RB et al (2001) Pharmacopoeial quality of drugs supplied by Nigerian pharmacies. Lancet 357(9272):1933-1936. https:// doi.org/10.1016/S0140-6736(00)05065-0

16. Ehigiator O, Azodo C, Ehikhamenor E (2011) Self-medication with antibiotics among Nigerian dental students. Tanzania Dent J 16(2):48-53. https://doi.org/10.4314/tdj.v16i2.69869

17. WHO (2000) Guidelines for the regulatory assessment of medicinal products for use in self-medication. World Health Organization, Geneva https://apps.who.int/iris/handle/10665/66154

18. Adu-Sarkodie YA (1997) Antimicrobial self medication in patients attending a sexually transmitted diseases clinic. Int J STD AIDS 8(7):456-458. https://doi.org/10.1258/0956462971920343

19. Donkor E et al (2012) Self-medication practices with antibiotics among tertiary level students in accra, Ghana: a cross-sectional 
study. Int J Environ Res Public Health 9(10):3519-3529. https:// doi.org/10.3390/ijerph9103519

20. Kümmerer K (2009) Antibiotics in the aquatic environment-a review-part I. Chemosphere 75(4):417-434. Pergamon. https ://doi.org/10.1016/j.chemosphere.2008.11.086

21. Mitema ES, Kikuvi GM (2004) Surveillance of the overall use of antimicrobial drugs in humans over a 5 year period (1997-2001) in Kenya. J Antimicrob Chemother 54(5):966-967. https://doi. org/10.1093/jac/dkh433

22. Schar D et al (2018) Surveillance of antimicrobial consumption in animal production sectors of low-and middle-income countries: optimizing use and addressing antimicrobial resistance. PLoS Med 15(3):e1002521. https://doi.org/10.1371/journ al.pmed. 1002521

23. Liu $\mathrm{F}$ et al (2018) Simultaneous screening and determination of eight tetracycline antibiotics illegally adulterated in herbal preparations using HPLC-DAD combined with LC-MS-MS. Chromatographia 81(2):303-314. https://doi.org/10.1007/s1033 7-017-3450-8

24. Luo W et al (1997) Simultaneous determination of amoxicillin and ampicillin in bovine milk by HPLC with fluorescence detection. J Agric Food Chem 45(4):1264-1268. https://doi. org/10.1021/jf960739l

25. Takeba K et al (1998) Simultaneous determination of betalactam antibiotics in milk by ion-pair liquid chromatography. J Chromatogr A 812(1-2):205-211. https://doi.org/10.1016/s0021 $-9673(97) 01261-2$

26. Tyczkowska KL et al (1994) Simultaneous multiresidue analysis of beta-lactam antibiotics in bovine milk by liquid chromatography with ultraviolet detection and confirmation by electrospray mass spectrometry. J AOAC Int 77(5):1122-1131 http://www. ncbi.nlm.nih.gov/pubmed/7950414. Accessed 5 Mar 2020

27. Voigt $\mathrm{AM}$ et al (2020) Liquid chromatography-tandem mass spectrometry as a fast and simple method for the determination of several antibiotics in different aqueous matrices. Environ Chem 17(1):54. https://doi.org/10.1071/EN19115

28. Kumar A et al (2008) Gradient HPLC of antibiotics in urine, ground water, chicken muscle, hospital wastewater, and pharmaceutical samples using C-18 and RP-amide columns. J Sep Sci 31(2):294-300. https://doi.org/10.1002/jssc.200700373

29. Shintani $H$ (2013) Liquid-liquid extraction vs solid phase extraction in biological fluids. Int J Clin Pharmacol Toxicol 2(2):1

30. Li K, Rivory L, Clarke S (2006) Solid-phase extraction (SPE) techniques for sample preparation in clinical and pharmaceutical analysis: a brief overview. Curr Pharm Anal 2(2):95-102. https:// doi.org/10.2174/157341206776819346

31. Taylor PJ (2005) Matrix effects: the Achilles heel of quantitative high-performance liquid chromatography-electrospray-tandem mass spectrometry. Clin Biochem 38(4):328-334. https://doi. org/10.1016/j.clinbiochem.2004.11.007

32. ICH (2019) ICH M10 guideline on bioanalytical method validation. www.ema.europa.eu/contact. Accessed 5 Mar 2020
33. Kaza M et al (2019) Bioanalytical method validation: new FDA guidance vs. EMA guideline. Better or worse? J Pharm Biomed Anal 165:381-385. https://doi.org/10.1016/j.jpba.2018.12.030

34. Bekoe SO et al (2014) Determination of thirteen antibiotics in drug products-a new LC-MS/MS tool for screening drug product quality. Anal Methods 6(15):5847-5855. https://doi. org/10.1039/c4ay00748d

35. European Commission (2002) Commission decision of 12 August 2002 implementing Council Directive 96/23/EC concerning the performance of analytical methods and the interpretation of results. Off J Eur Commun L 221/8:8-36. https://doi.org/10.1017/ CBO9781107415324.004

36. Adriaenssens $\mathrm{N}$ et al (2011) European Surveillance of Antimicrobial Consumption (ESAC): outpatient quinolone use in Europe (1997-2009). J Antimicrob Chemother 66(Suppl 6):47-56. https ://doi.org/10.1093/jac/dkr457

37. Chandy SJ et al (2013) Patterns of antibiotic use in the community and challenges of antibiotic surveillance in a lower-middleincome country setting: a repeated cross-sectional study in Vellore, South India. J Antimicrob Chemother 68(1):229-236. https ://doi.org/10.1093/jac/dks355

38. Ferech $\mathrm{M}$ et al (2006) European Surveillance of Antimicrobial Consumption (ESAC): outpatient antibiotic use in Europe. J Antimicrob Chemother 58(2):401-407. https://doi.org/10.1093/jac/ dkl188

39. Afari-Asiedu S et al (2018) To sell or not to sell; The differences between regulatory and community demands regarding access to antibiotics in rural Ghana. J Pharm Policy Pract 11(1):1-10. https://doi.org/10.1186/s40545-018-0158-6

40. Gu J et al (2015) Use of antibiotics by urban and rural residents in Heilongjiang Province, China: cross-sectional study. Trop Med Int Health 20(12):1815-1822. https://doi.org/10.1111/tmi.12602

41. Simoens $S$ (2011) Factors affecting the cost effectiveness of antibiotics. Chemother Res Pract 2011:1-6. https://doi. org/10.1155/2011/249867

42. Azanu D et al (2018) Occurrence and risk assessment of antibiotics in water and lettuce in Ghana. Sci Total Environ 622-623:293305. https://doi.org/10.1016/j.scitotenv.2017.11.287

43. Darko $\mathrm{G}$ et al (2017) Veterinary antibiotics in dairy products from Kumasi, Ghana. Cogent Chem 3(1). Edited by R. Bernardino. https://doi.org/10.1080/23312009.2017.1343636

44. $\mathrm{MOH}(2010)$ Essential medicines list, 6th edn. Ministry of Health. www.ghndp.org

45. STG (2010) Standard treatment guidelines, 6th edn. Ghana National Drugs Programme, Accra www.ghndp.org

46. Suthar AB, Coggin W, Raizes E (2019) Antimicrobial resistance and substandard and falsified medicines: the case of HIV/AIDS. $\mathrm{J}$ Infect Dis 219(4):672-673 https://academic.oup.com/jid/artic le/219/4/672/5126519. Accessed 5 Mar 2020

Publisher's Note Springer Nature remains neutral with regard to jurisdictional claims in published maps and institutional affiliations. 